\title{
The Number of Spanning Trees of the Cartesian Product of Regular Graphs
}

\author{
Mei-Hui $\mathrm{Wu}^{1}$ and Long-Yeu Chung ${ }^{2}$ \\ ${ }^{1}$ The Center of General Education, Information Technology Section, Chia Nan University of Pharmacy and Science, \\ Tainan 71710, Taiwan \\ ${ }^{2}$ Department of Applied Informatics and Multimedia, Chia Nan University of Pharmacy and Science, \\ Tainan 71710, Taiwan
}

Correspondence should be addressed to Long-Yeu Chung; chungly1@mail.chna.edu.tw

Received 18 March 2014; Accepted 7 May 2014; Published 14 July 2014

Academic Editor: Her-Terng Yau

Copyright (C) 2014 M.-H. Wu and L.-Y. Chung. This is an open access article distributed under the Creative Commons Attribution License, which permits unrestricted use, distribution, and reproduction in any medium, provided the original work is properly cited.

\begin{abstract}
The number of spanning trees in graphs or in networks is an important issue. The evaluation of this number not only is interesting from a mathematical (computational) perspective but also is an important measure of reliability of a network or designing electrical circuits. In this paper, a simple formula for the number of spanning trees of the Cartesian product of two regular graphs is investigated. Using this formula, the number of spanning trees of the four well-known regular networks can be simply taken into evaluation.
\end{abstract}

\section{Introduction}

In this paper, we deal with simple undirected graphs having no self-loop or multiple edges and consider the Cartesian product of two regular graphs only. It is well known that, for designing large-scale interconnection networks, the Cartesian product is an important method to obtain large networks from smaller ones, with a number of parameters that can be easily calculated from the corresponding parameters for those small initial graphs. The Cartesian product preserves many nice properties such as regularity, transitivity, super edge-connectivity, and super point-connectivity of the initial regular graphs [1-6]. In fact, many well-known networks can be constructed by the Cartesian products of simple regular graphs, for example, Boolean $n$-cube networks, hypercube networks, and lattice networks.

Alternatively, the study of the number of spanning trees in a graph has a long history and has been very active because the problem has different practical applications in different fields. For example, the number characterizes the reliability of a network and, in physics, designing electrical circuits, analyzing energy of masers, and investigating the possible particle transitions [7-10]. The larger degree of points a network has, the more I/O ports and edges are needed and the more cost is required.

The number of spanning trees of some special network has been taken into evaluation [11-20]. Recently, some authors derived results about the counting where the number of spanning trees can be found from [21-29]. However, the study for spanning trees of the Cartesian product of regular graphs remains an open and important invariant.

The number of spanning trees of Boolean $n$-cube networks, lattice networks, and generalized Boolean $n$-cube networks has been taken into account $[13,17,18]$; these networks belong to the class of networks $Q_{n}$ with two regular graphs $Q_{1}$ and $G$ which is defined recursively by $Q_{n}=Q_{n-1} \times$ $G$ for $n \geq 2$. In this paper, we will present the formula of the number of spanning trees of the Cartesian product of regular graphs. Using this present formula, the main results in $[13,17,18]$ can be obtained much more simply and will be extended. 


\section{The Number of Spanning Trees}

Definition 1. Let $G$ be a graph with $n$ points labeled $1,2, \ldots, n$. The adjacency matrix of $G, A(G)$, is an $n \times n$ matrix with the $i$ th row and $j$ th column entry given by

$$
[A(G)]_{i j}= \begin{cases}1 & \text { if points } i \text { and } j \text { are adjacent } \\ 0 & \text { ortherwise. }\end{cases}
$$

The Kirchhoff matrix of $G, H(G)$, is equal to $D(G)-A(G)$, where $D(G)$ is an $n \times n$ diagonal matrix whose diagonal entries are the degree of point $n$ and $A(G)$ is the adjacency matrix. Thus the $i$ th row and $j$ th column entry is given by

$$
\begin{aligned}
& {[H(G)]_{i j}} \\
& \quad= \begin{cases}\operatorname{deg}(i) & \text { if } i=j \\
-1 & \text { if } i \neq j \text { and points } i \text { and } j \text { are adjacent } \\
0 & \text { ortherwise. }\end{cases}
\end{aligned}
$$

Lemma 2 (see [30]). If $G$ is a graph on $n$ points with Kirchhoff matrix $H(G)$ and $H_{i j}(G)$ is the submatrix of $H(G)$ obtained by removing the ith row and jth column then the number of spanning trees of $G, t(G)$, is any cofactor of $H(G)$. That is, $t(G)=(-1)^{i+j} \operatorname{det}\left(H_{i j}(G)\right)$.
Lemma 3. If $A$ is an $n \times n$ triangulable matrix, which has $n$ eigenvalues, then the sum of product of any $n-1$ eigenvalues of $A$ is the sum of all principal minors of $A$.

Proof. Let $p(\lambda)$ be the character polynomial of $A$ and $\lambda_{1}, \lambda_{2}, \ldots, \lambda_{n}$ are $n$ eigenvalues of $A$. Then

$$
p(\lambda)=\left(\lambda-\lambda_{1}\right)\left(\lambda-\lambda_{2}\right) \cdots\left(\lambda-\lambda_{n}\right)
$$

From (3), we obtain the following.

(a) The coefficient of $\lambda=(-1)^{n-1}$. (the sum of product of any $n-1$ eigenvaules of $A$ ).

On the other hand,

$$
\begin{aligned}
p(\lambda) & =\operatorname{det}(\lambda I-A) \\
& =\left|\begin{array}{cccc}
\lambda^{(1)}-a_{11} & -a_{12} & \cdots & -a_{1 n} \\
-a_{21} & \lambda^{(2)}-a_{22} & \cdots & -a_{2 n} \\
\vdots & \vdots & \ddots & \vdots \\
-a_{21} & -a_{21} & \cdots & \lambda^{(n)}-a_{n n}
\end{array}\right|,
\end{aligned}
$$

where $A=\left[a_{i j}\right]$ and $\lambda^{(i)}=\lambda$ for $i=1,2, \ldots, n$.

So we only need to prove that the coefficient of $\lambda$ in $\operatorname{det}(\lambda I-A)$ is the sum of all principal minors of $A$. Let $A_{i i}$ denote the principal minor of $A$ obtained by removing the $i$ th row and $i$ th column from $A$.

By (4), we obtain the following:

$$
\begin{aligned}
& \text { the coefficient of } \lambda^{(1)}=(-1)^{n-1} A_{11} \quad\left(\text { taking } \lambda^{(2)}=\lambda^{(3)}=\cdots=\lambda^{(n)}=0\right) \\
& \text { the coefficient of } \lambda^{(2)}=(-1)^{n-1} A_{22} \quad\left(\text { taking } \lambda^{(1)}=\lambda^{(3)}=\lambda^{(4)} \cdots=\lambda^{(n)}=0\right) \\
& \vdots \\
& \text { the coefficient of } \lambda^{(n)}=(-1)^{n-1} A_{n n}\left(\text { taking } \lambda^{(1)}=\lambda^{(2)}=\cdots=\lambda^{(n-1)}=0\right) .
\end{aligned}
$$

(b) So the coefficient of $\lambda=\sum_{i=1}^{n}$ the coefficient of $\lambda^{(i)}=$ $(-1)^{n-1}\left(A_{11}+A_{22}+\cdots+A_{n n}\right)$.

Hence the theorem is proved due to (a) and (b).

Since a real symmetric matrix is with the property that the sum of its rows (and its columns) is zero, the rank of $H(G) \leq$ $n-1$. So 0 is the smallest eigenvalue. We write the eigenvalues of $H(G)$ as an ordered list:

$$
0=\lambda_{0}(G) \leq \lambda_{1}(G) \leq \lambda_{2}(G) \cdots \leq \lambda_{n-1}(G) .
$$

The main result in Kelmans and Chelnokov [31] can also be obtained by the following method.

Lemma 4 (see [31]). If the eigenvalues of the Kirchhoff matrix $H(G)$ of the $n$ points graph $G$ are $0=\lambda_{0}(G) \leq \lambda_{1}(G) \leq$ $\lambda_{2}(G) \cdots \leq \lambda_{n-1}(G)$ then $t(G)$, the number of spanning trees of $G$, is given by

$$
t(G)=\frac{1}{n} \prod_{i=1}^{n-1} \lambda_{i}(G)
$$

Proof. By Lemmas 2 and 3,

$\prod_{i=1}^{n-1} \lambda_{i}(G)$

$=$ the sum of product of any $n-1$ eigenvaules of $H(G)$

= the sum of all principal minors of $H(G)=n \cdot t(G)$.

Hence $t(G)=(1 / n) \prod_{i=1}^{n-1} \lambda_{i}(G)$.

Lemma 5. Let the eigenvalues of the adjacency matrix $A(G)$ of the regular graph $G$ be written by $u_{1} \leq u_{2} \cdots \leq u_{n-1} \leq u_{n}=n$, where $r$ is the degree of the regular graph $G$; then, the number of spanning trees of $G$ is given by

$$
t(G)=\frac{1}{n} \prod_{i=1}^{n-1}\left(r-u_{i}\right)
$$


Proof. We know $H(G)=r I_{n}-A(G)$, where $I_{n}$ is the identity $n \times n$ matrix. Since $u_{i}$ is the eigenvalue of $A(G)$ for $i=$ $1,2, \ldots, n-1$, there exists eigenvector $x_{i}$ for $i=1,2, \ldots, n-1$, such that $A(G) x_{i}=u_{i} x_{i}$. So $\left(r I_{n}-H(G)\right) x_{i}=u_{i} x_{i}, r I_{n} x_{i}-$ $H(G) x_{i}=u_{i} x_{i}, r x_{i}-H(G) x_{i}=u_{i} x_{i}$, and $H(G) x_{i}=r x_{i}-u_{i} x_{i}$.

We obtain $H(G) x_{i}=\left(r-u_{i}\right) x_{i}$. Thus $r-u_{i}$ is the eigenvalue of $H(G)$ for $i=1,2, \ldots, n-1$.

Hence the lemma is proved by Lemma 4.

\section{Cartesian Product and Kronecker Product}

Definition 6. Let $G=(N, E)$ denote a connected graph with $N$ set of all points and $E$ set of all edges in $G$ and let $\{u, v\}$ denote edge joining points $u$ and $v$. Let $G_{i}=\left(N_{i}, E_{i}\right)$ for $i=1,2$; the Cartesian product of $G_{1}$ and $G_{2}$ is defined by $G_{1} \times G_{2}=(N, E)$, where $N=N_{1} \times N_{2}, E=E_{1} \times E_{2}$, and $\left\{\left(u_{1}, v_{1}\right),\left(u_{2}, v_{2}\right)\right\} \in E$ if and only if $u_{1}=u_{2}$ and $\left\{v_{1}, v_{2}\right\} \in E_{2}$ or $v_{1}=v_{2}$ and $\left\{u_{1}, u_{2}\right\} \in E_{1}$.

Definition 7 (see [32]). Let $B=\left[b_{i j}\right]$ be an $n \times n$ matrix and $C$ an $m \times m$ matrix; then, the Kronecker product $B \times{ }_{K} C$ is defined as the $m n \times m n$ matrix with block description

$$
\left[\begin{array}{cccc}
b_{11} C & b_{12} C & \cdots & b_{1 n} C \\
b_{21} C & b_{22} C & \cdots & b_{2 n} C \\
\vdots & \vdots & \ddots & \vdots \\
b_{n 1} C & b_{n 2} C & \cdots & b_{n n} C
\end{array}\right]
$$

The Kronecker sum is defined by $B+{ }_{K} C=B \times{ }_{K} I_{m}+I_{n} \times{ }_{K} C$, where $I_{k}$ is the $k \times k$ identity matrix for $k=m, n$. Let $M$ be an $m n \times m n$ matrix. $M$ can be partitioned into $n^{2}$ blocks which are denoted by $B_{\alpha \beta}$ for $\alpha=1,2, \ldots, n$ and $\beta=1,2, \ldots, n$. That is,

$$
M=\left[\begin{array}{cccc}
B_{11} & B_{12} & \cdots & B_{1 n} \\
B_{21} & B_{22} & \cdots & B_{n 1} \\
\vdots & \vdots & \ddots & \vdots \\
B_{n 1} & B_{n 2} & \cdots & B_{n n}
\end{array}\right]
$$

where $B_{\alpha \beta}$ is the $m \times m$ matrix for $\alpha=1,2, \ldots, n$ and $\beta=$ $1,2, \ldots, n$. $M$ is called an $n \times n \quad(m \times m)$ block matrix.

Lemma 8. If $A$ is an $n \times n$ matrix and $B, C$ are $m \times m$ matrices then

(1) $A \times_{K}(B+C)=A \times_{K} B+A \times_{K} C$,

(2) $(B+C) \times_{K} A=B \times_{K} A+C \times_{K} A$.
Proof. The lemma is easily obtained.

Lemma 9. If the products $A B$ and $C D$ are defined then $(A B) \times_{K}(C D)=\left(A \times_{K} C\right)\left(B \times_{K} D\right)$.

Proof. Let $A=\left[a_{i j}\right]$ be an $m \times n$ matrix and $B=\left[b_{i j}\right]$ an $n \times p$ matrix

$$
\begin{aligned}
\left(A \times_{K} C\right)\left(B \times{ }_{K} D\right) & =\left[\begin{array}{ccc}
a_{11} C & \cdots & a_{1 n} C \\
\vdots & \ddots & \vdots \\
a_{m 1} C & \cdots & a_{m n} C
\end{array}\right]\left[\begin{array}{ccc}
b_{11} D & \cdots & b_{1 p} D \\
\vdots & \ddots & \vdots \\
b_{n 1} D & \cdots & b_{n p} D
\end{array}\right] \\
& =\left[\begin{array}{cccc}
\sum_{k=1}^{n} a_{1 k} b_{k 1} C D & \cdots & \sum_{k=1}^{n} a_{1 k} b_{k p} C D \\
\vdots & \ddots & \vdots \\
\sum_{k=1}^{n} a_{m k} b_{k 1} C D & \cdots & \sum_{k=1}^{n} a_{m k} b_{k p} C D
\end{array}\right] \\
& =(A B) \times{ }_{K}(C D) .
\end{aligned}
$$

Lemma 10. If $P$ and $Q$ are invertible then $\left(P \times_{K} Q\right)^{-1}=$ $P^{-1} \times{ }_{K} Q^{-1}$.

Proof. Consider

$$
\begin{aligned}
\left(P \times_{K} Q\right)\left(P^{-1} \times_{K} Q^{-1}\right) & =\left(P P^{-1}\right) \times_{K}\left(Q Q^{-1}\right) \\
& =I_{m} \times_{K} I_{n}=I_{m n},
\end{aligned}
$$

where $P$ is $m \times m$ and $Q$ is $n \times n$.

\section{The Number of Spanning Trees of the Cartesian Product of Regular Graphs}

Lemma 11. If the points of $G_{1}$ and $G_{2}$ are labeled by $u_{1}, u_{2}, \ldots u_{n}$ and $v_{1}, v_{2}, \ldots, v_{m}$, respectively, and points of $G_{1} \times$ $G_{2}$ are ordered lexicographically, that is, the label of $\left(u_{i}, v_{j}\right)$ is smaller than that of $\left(u_{k}, v_{l}\right)$ if and only if $i<k$ and $j<l$, then $A\left(G_{1} \times G_{2}\right)=A\left(G_{1}\right)+_{K} A\left(G_{2}\right)$.

Proof. Since $A\left(G_{1} \times G_{2}\right)$ is an $m n \times m n$ matrix, $A\left(G_{1} \times G_{2}\right)$ is an $n \times n(m \times m)$ block matrix. By the definition of $G_{1} \times G_{2}$, we describe

$$
A\left(G_{1} \times G_{2}\right)=\left[\begin{array}{cccccc}
A\left(G_{2}\right) & {\left[A\left(G_{1}\right)\right]_{12} I_{m}} & {\left[A\left(G_{1}\right)\right]_{13} I_{m}} & \cdots & {\left[A\left(G_{1}\right)\right]_{1, n-1} I_{m}} & {\left[A\left(G_{1}\right)\right]_{1, n} I_{m}} \\
{\left[A\left(G_{1}\right)\right]_{21} I_{m}} & A\left(G_{2}\right) & {\left[A\left(G_{1}\right)\right]_{23} I_{m}} & \cdots & {\left[A\left(G_{1}\right)\right]_{2, n-1} I_{m}} & {\left[A\left(G_{1}\right)\right]_{2, n} I_{m}} \\
\vdots & \vdots & \vdots & & \vdots & \vdots \\
{\left[A\left(G_{1}\right)\right]_{n-1,1} I_{m}} & {\left[A\left(G_{1}\right)\right]_{n-1,2} I_{m}} & {\left[A\left(G_{1}\right)\right]_{n-1,3} I_{m}} & \cdots & A\left(G_{2}\right) & {\left[A\left(G_{1}\right)\right]_{n-1, n} I_{m}} \\
{\left[A\left(G_{1}\right)\right]_{n, 1} I_{m}} & {\left[A\left(G_{1}\right)\right]_{n, 2} I_{m}} & {\left[A\left(G_{1}\right)\right]_{n, 3} I_{m}} & \cdots & {\left[A\left(G_{1}\right)\right]_{n, n-1} I_{m}} & A\left(G_{2}\right)
\end{array}\right],
$$


where $\left[A\left(G_{1}\right)\right]_{i j}$ is the $(i, j)$ entry of the adjacent matrix $A\left(G_{1}\right)$ of $G_{1}$ and $A\left(G_{2}\right)$ is the $m \times m$ adjacent matrix of $G_{2}$. We know
$I_{n} \times{ }_{K} A\left(G_{2}\right)$ is an $m n \times m n$ matrix; it can be described as an $n \times n(m \times m)$ block matrix

$$
I_{n} \times{ }_{K} A\left(G_{2}\right)=\left[\begin{array}{cccccc}
A\left(G_{2}\right) & 0_{m \times m} & 0_{m \times m} & \cdots & 0_{m \times m} & 0_{m \times m} \\
0_{m \times m} & A\left(G_{2}\right) & 0_{m \times m} & \cdots & 0_{m \times m} & 0_{m \times m} \\
\vdots & \vdots & \vdots & \cdots & \vdots & \vdots \\
0_{m \times m} & 0_{m \times m} & 0_{m \times m} & \cdots & A\left(G_{2}\right) & 0_{m \times m} \\
0_{m \times m} & 0_{m \times m} & 0_{m \times m} & \cdots & 0_{m \times m} & A\left(G_{2}\right)
\end{array}\right]
$$

$$
A\left(G_{1}\right) \times_{K} I_{m}=\left[\begin{array}{cccccc}
0_{m \times m} & {\left[A\left(G_{1}\right)\right]_{12} I_{m}} & {\left[A\left(G_{1}\right)\right]_{13} I_{m}} & \cdots & {\left[A\left(G_{1}\right)\right]_{1, n-1} I_{m}} & {\left[A\left(G_{1}\right)\right]_{1, n} I_{m}} \\
{\left[A\left(G_{1}\right)\right]_{21} I_{m}} & 0_{m \times m} & {\left[A\left(G_{1}\right)\right]_{23} I_{m}} & \cdots & {\left[A\left(G_{1}\right)\right]_{2, n-1} I_{m}} & {\left[A\left(G_{1}\right)\right]_{2, n} I_{m}} \\
\vdots & \vdots & \vdots & & \vdots & \vdots \\
{\left[A\left(G_{1}\right)\right]_{n-1,1} I_{m}} & {\left[A\left(G_{1}\right)\right]_{n-1,2} I_{m}} & {\left[A\left(G_{1}\right)\right]_{n-1,3} I_{m}} & \cdots & 0_{m \times m} & {\left[A\left(G_{1}\right)\right]_{n-1, n} I_{m}} \\
{\left[A\left(G_{1}\right)\right]_{n, 1} I_{m}} & {\left[A\left(G_{1}\right)\right]_{n, 2} I_{m}} & {\left[A\left(G_{1}\right)\right]_{n, 3} I_{m}} & \cdots & {\left[A\left(G_{1}\right)\right]_{n, n-1} I_{m}} & 0_{m \times m}
\end{array}\right] .
$$

Clearly $A\left(G_{1} \times G_{2}\right)=A\left(G_{1}\right) \times_{K} I_{m}+I_{n} \times_{K} A\left(G_{2}\right)=A\left(G_{1}\right)+_{K}$ $A\left(G_{2}\right)$.

Lemma 12. Let $G_{i}$ be the regular graph of degree $r_{i}$ for $i=1,2$; then, the degree of $G_{1} \times G_{2}$ is $r_{1}+r_{2}$. If the number of the points of $G_{1}$ (resp., $G_{2}$ ) is $n$ (resp., $m$ ) and the points of $G_{1} \times G_{2}$ are ordered lexicographically then $H\left(G_{1} \times G_{2}\right)=H\left(G_{1}\right){ }_{K} H\left(G_{2}\right)$.

Proof. By Lemmas 8 and 11,

$$
\begin{aligned}
H\left(G_{1} \times G_{2}\right)= & \left(r_{1}+r_{2}\right) I_{m n}-A\left(G_{1} \times G_{2}\right) \\
= & \left(r_{1}+r_{2}\right) I_{m n}-\left(A\left(G_{1}\right){ }_{K} A\left(G_{2}\right)\right) \\
= & \left(r_{1}+r_{2}\right) I_{m n}-\left(A\left(G_{1}\right) \times_{K} I_{m}+I_{n} \times_{K} A\left(G_{2}\right)\right) \\
= & \left(r_{1} I_{m n}-I_{n} \times{ }_{K} A\left(G_{2}\right)\right) \\
& +\left(r_{2} I_{m n}-A\left(G_{1}\right) \times_{K} I_{m}\right) \\
= & \left(I_{n} \times_{K} r_{1} I_{m}-I_{n} \times{ }_{K} A\left(G_{2}\right)\right) \\
& +\left(r_{2} I_{n} \times_{K} I_{m}-A\left(G_{1}\right) \times_{K} I_{m}\right) \\
= & I_{n} \times_{K}\left(r_{1} I_{m}-A\left(G_{2}\right)\right)+\left(r_{2} I_{n}-A\left(G_{1}\right)\right) \times_{K} I_{m} \\
= & I_{n} \times{ }_{K} H\left(G_{2}\right)+H\left(G_{1}\right) \times_{K} I_{m} \\
= & H\left(G_{1}\right)+{ }_{K} H\left(G_{2}\right),
\end{aligned}
$$

where $I_{m m}$ is the $m n \times m n$ identity matrix.

Lemma 13. If $A$ and $B$ are triangulable matrices then the eigenvalues of $A+{ }_{k} B$ are given by $\alpha+\beta$, respectively, as $\alpha$ and $\beta$ vary through the eigenvalues of $A$ and $B$. where $0_{m \times m}$ is the $m \times m$ zero matrix. Since $A\left(G_{1}\right) \times_{K} I_{m}$ is an $m n \times m n$ matrix, it can be described as an $n \times n(m \times m)$ block matrix
Proof. Since $A$ and $B$ are triangulable, there exist invertible matrices $Q$ and $P$ such that $A_{1}=Q A Q^{-1}$ and $B_{1}=P B P^{-1}$ are upper triangular. If $A$ and $B$ are $n \times n$ and $m \times m$ matrices, respectively, by Lemmas 9 and 10,

$$
\begin{aligned}
A_{1}+{ }_{k} B_{1}= & A_{1} \times_{K} I_{m}+I_{n} \times_{K} B_{1} \\
= & \left(Q A Q^{-1}\right) \times_{K}\left(P I_{m} P^{-1}\right) \\
& +\left(Q I_{n} Q^{-1}\right) \times_{K}\left(P B P^{-1}\right) \\
= & \left(Q \times_{K} P\right)\left(A Q^{-1} \times_{K} I_{m} P^{-1}\right) \\
& +\left(Q \times_{K} P\right)\left(I_{n} Q^{-1} \times_{K} B P^{-1}\right) \\
= & \left(Q \times_{K} P\right)\left(A \times_{K} I_{m}\right)\left(Q^{-1} \times_{K} P^{-1}\right) \\
& +\left(Q \times{ }_{K} P\right)\left(I_{n} \times_{K} B\right)\left(Q^{-1} \times_{K} P^{-1}\right) \\
= & \left(Q \times_{K} P\right)\left[\left(A \times_{K} I_{m}\right)+\left(I_{n} \times_{K} B\right)\right]\left(Q \times_{K} P^{-1}\right) \\
= & \left(Q \times{ }_{K} P\right)\left[\left(A \times_{K} I_{m}\right)+\left(I_{n} \times_{K} B\right)\right]\left(Q \times_{K} P\right)^{-1} \\
= & \left(Q \times{ }_{K} P\right)\left(A{ }_{k} B\right)\left(Q \times_{K} P\right)^{-1} .
\end{aligned}
$$

So $A+{ }_{k} B$ is similar to $A_{1}+{ }_{k} B_{1}$ and they have the same eigenvalues. Obviously $A_{1}+{ }_{k} B_{1}=A_{1} \times{ }_{K} I_{m}+I_{n} \times{ }_{K} B_{1}$ is upper triangular with diagonal entries given by $\alpha+\beta$, respectively, as $\alpha$ and $\beta$ vary through the eigenvalues of $A_{1}$ and $B_{1}$. Hence the eigenvalues of $A+{ }_{k} B$ are $\alpha+\beta$, respectively, as $\alpha$ and $\beta$ vary through the eigenvalues of $A$ and $B$. 
Theorem 14. Let $G_{1}$ and $G_{2}$ be the regular graphs with degrees $m$ and $n$, respectively. If the eigenvalues of the adjacency matrix $A\left(G_{1}\right)$ are written as $\alpha_{1} \leq \alpha_{2} \cdots \leq \alpha_{n-1} \leq \alpha_{m}=m$ and the eigenvalues of the adjacency matrix $A\left(G_{2}\right)$ are written as $\beta_{1} \leq \beta_{2} \cdots \leq \beta_{n-1} \leq \beta_{n}=n$, then the number of spanning trees of the Cartesian product $G_{1} \times G_{2}$ is

$$
t\left(G_{1} \times G_{2}\right)=\frac{1}{m n} \prod_{i, j}\left[(m+n)-\left(\alpha_{i}+\beta_{j}\right)\right],
$$

where $i$ and $j$ satisfy $(i, j) \in\{1,2, \ldots, m\} \times\{1,2, \ldots, n\}-$ $\{(m, n)\}$.

Proof. We know $G_{1} \times G_{2}$ has $m n$ points and the degree of $G_{1} \times$ $G_{2}$ is $m+n$. By Lemma $11, A\left(G_{1} \times G_{2}\right)=A\left(G_{1}\right)+{ }_{K} A\left(G_{2}\right)$. By Lemma 13, the eigenvalues of $A\left(G_{1} \times G_{2}\right)$ are $\alpha_{i}+\beta_{j}$ for $i=$ $1,2, \ldots, m$ and $j=1,2, \ldots, n$. The result follows by Lemma 5 .

Theorem 15. If $G_{1}$ and $G_{2}$ are the regular graph of degrees $m$ and $n$, respectively, the eigenvalues of the Kirchhoff matrix $H\left(G_{1}\right)$ are written as $0=\lambda_{0} \leq \lambda_{1} \cdots \leq \lambda_{m-1}$, and the eigenvalues of the Kirchhoff matrix $H\left(G_{2}\right)$ are written as $0=$ $\gamma_{0} \leq \gamma_{1} \cdots \leq \gamma_{n-1}$, then the number of spanning trees of the Cartesian product of $G_{1}$ and $G_{2}$ is

$$
t\left(G_{1} \times G_{2}\right)=\frac{1}{m n} \prod_{i, j}\left(\lambda_{i}+\gamma_{j}\right),
$$

where $i$ and $j$ satisfy $(i, j) \in\{0,1, \ldots, m-1\} \times\{0,1, \ldots, n-$ $1\}-\{(0,0)\}$.

Proof. By Lemma 12, $H\left(G_{1} \times G_{2}\right)=H\left(G_{1}\right){ }_{K} H\left(G_{2}\right)$. By Lemma 13, the eigenvalues of $H\left(G_{1} \times G_{2}\right)$ are $\lambda_{i}+\gamma_{j}$ for $i=0,1, \ldots, m-1$ and $j=0,1, \ldots, n-1$. Hence by Lemma 4 , the result follows.

\section{The Number of Spanning Trees of the $r^{n}$-Lattice Network}

Definition 16 (see $[17,18])$. The $r^{n}$-lattice networks $R(r, n)$ are defined as

$$
R(r, n)= \begin{cases}K_{r} & \text { for } n=1 \\ R(r, n-1) \times K_{r} & \text { for } n \geq 2,\end{cases}
$$

where $K_{r}$ is a complete graph of $r$ points.

When $r=2, R(2, n)$ is well known, the Boolean $n$-cube network.

We denote $R(r, n)=K_{r}^{(1)} \times K_{r}^{(2)} \times \cdots \times K_{r}^{(n)}$.

Lemma 17. The eigenvalues of $H\left(K_{r}\right)$ are $r$ with multiplicity $r-1$ and 0 with multiplicity 1.

Proof. Since $H\left(K_{r}\right)=r I_{r}-J_{r}$, where $J_{r}$ is the matrix of all ones, letting $P_{K_{r}}(\lambda)$ be the character polynomial of $H\left(K_{r}\right)$, we obtain by Gaussian elimination

$$
\begin{aligned}
P_{K_{r}}(\lambda) & =\lambda\left|\begin{array}{ccccc}
1 & 1 & 1 & \ldots & 1 \\
0 & \lambda-r & -1 & \ldots & \ldots \\
0 & 0 & \lambda-r & \ldots & \ldots \\
\vdots & \vdots & \vdots & \ddots & \ldots \\
0 & 0 & 0 & 0 & \lambda-r
\end{array}\right| \\
& =\lambda(\lambda-r)^{r-1} .
\end{aligned}
$$

Hence the result follows.

Lemma 18. If the distinct eigenvalues of the Kirchhoff matrix $H(R(r, n))$ are $0=\lambda_{0} \leq \lambda_{1} \cdots \leq \lambda_{n-1} \leq \lambda_{n}$ then

$$
\begin{aligned}
\lambda_{0} & =\text { with multiplicity } C(n, n)=1 \\
\lambda_{1} & =r \text { with multiplicity }(r-1) C(n, n-1) \\
\lambda_{2} & =2 r \text { with multiplicity }(r-1)^{2} C(n, n) \\
\vdots & \\
\lambda_{n-1} & =(n-1) r \text { with multiplicity }(r-1)^{n-1} C(n, 1) \\
\lambda_{n} & =n r \text { with multiplicity }(r-1)^{n} C(n, 0),
\end{aligned}
$$

where $C(n, r)=n ! / r !(n-r) !$

Proof. Since $R(r, n)=K_{r}^{(1)} \times K_{r}^{(2)} \times \cdots \times K_{r}^{(n)}$ and by Lemma 13, we obtain each of eigenvalues of $H(R(r, n))=\sum_{i=1}^{n}$ one of eigenvalues of $H\left(K_{r}^{(i)}\right)$.

Hence if we take the eigenvalue 0 of $H\left(K_{r}^{(i)}\right)$ for $i=$ $1,2, \ldots, n$ then $\lambda_{0}=0$ with multiplicity $C(n, n)=1$. If we take the eigenvalue $r$ of $H\left(K_{r}^{(l)}\right)$ for some one $l \in\{1,2, \ldots, n\}$ and the eigenvalue 0 of $H\left(K_{r}^{(i)}\right)$ for each $i \in\{1,2, \ldots, n\}-\{l\}$ then $\lambda_{1}=r$ with multiplicity $(r-1) C(n, n-1)$. If we take the eigenvalue $r$ of $H\left(K_{r}^{(l)}\right)$ and $H\left(K_{r}^{(m)}\right)$, respectively, for $l, m \in\{1,2, \ldots, n\}$ and the eigenvalue 0 of $H\left(K_{r}^{(i)}\right)$ for each $i \in\{1,2, \ldots, n\}-\{l, m\}$, then $\lambda_{2}=2 r$ with multiplicity $(r-1)^{2} C(n, n)$. We keep performing the same process. Hence the result follows.

The main theorem in $[17,18]$ can be obtained much more simply by Theorem 19 as follows. 
Theorem 19 (see [17]). The number of spanning trees of $R(r, n)$ is

$$
t(R(r, n))=r^{r^{n}-n-1} \prod_{i=2}^{n} i^{C(n, i)(r-1)^{i}} .
$$

Proof. Since the degree of $R(r, n)$ is $n(r-1)$, the number of points of $R(r, n)$ is $r^{n}$. By Lemma 18 and Theorem 15, we obtain

$$
\begin{aligned}
t(R(r, n)) & \\
= & \frac{1}{r^{n}} \cdot r^{C(n, n-1)(r-1)} \cdot(2 r)^{C(n, n-2)(r-1)^{2}} \\
& \cdot(3 r)^{C(n, n-3)(r-1)^{3}} \cdots(n r)^{C(n, 0)(r-1)^{n}}=\frac{1}{r^{n}} \\
& \cdot r^{C(n, n-1)(r-1)+C(n, n-2)(r-1)^{2}+C(n, n-3)(r-1)^{3}+\cdots+C(n, 0)(r-1)^{n}} \\
& \cdot \prod_{i=1}^{n} i^{C(n, n-i)(r-1)^{i}} \\
= & \frac{1}{r^{n}} \cdot r^{r^{n}-1} \cdot \prod_{i=1}^{n} i^{C(n, n-i)(r-1)^{i}} \\
= & r^{r^{n}-n-1} \prod_{i=2}^{n} i^{C(n, i)(r-1)^{i}} .
\end{aligned}
$$

Corollary 20 (see [18]). The number of spanning trees of the Boolean $n$-cube network $B_{n}$ is

$$
t\left(B_{n}\right)=2^{2^{n}-n-1} \prod_{i=2}^{n} i^{C(n, i)} .
$$

Proof. Since $B_{n}=R(2, n)$, by Theorem 15 , the result follows.

\section{The Number of Spanning Trees of the $2 \times 3 \cdots \times n$-Lattice Network}

Definition 21. The $2 \times 3 \cdots \times n$ lattice network $Q_{n}$ can be defined recursively by $Q_{2}=K_{2}$ and $Q_{n}=K_{n} \times Q_{n-1}$.

Thus $Q_{n}$ has $n$ ! points. We denote $Q_{n}=K_{2} \times K_{3} \times \cdots \times K_{n}$.

Theorem 22. The number of spanning trees of $Q_{n}$ is

$$
t\left(Q_{n}\right)=\frac{1}{n !} \prod_{i=1}^{n-1} \prod_{2 \leq r_{1}<r_{2}<\cdots<r_{i} \leq n}\left(\sum_{j=1}^{i} r_{j}\right)^{\prod_{j=1}^{i}\left(r_{j}-1\right)}
$$

Proof. Since the eigenvalues of $H\left(K_{r}\right)$ are $r$ with multiplicity $r-1$ and 0 with multiplicity 1 , the distinct $\sum_{i=0}^{n-1} C(n-1, i)=2^{n-1}$ eigenvalues of $Q_{n}$ are 0 and $\lambda_{i, r_{1}, r_{2}, \ldots, r_{i}}=\sum_{j=1}^{i} r_{j}$ with multiplicity $\left(r_{1}-1\right)\left(r_{2}-1\right) \cdots\left(r_{i}-1\right)$, where $r_{1}, r_{2}, \ldots, r_{i}$, satisfying $2 \leq r_{1}<r_{2}<\cdots<r_{i} \leq n$, $i=1,2, \ldots, n-1$, are nonzero eigenvalues of $H\left(K_{r_{1}}\right)$, $H\left(K_{r_{2}}\right), \ldots, H\left(K_{r_{i}}\right)$, respectively, and take zero eigenvalues for the remaining $H\left(K_{r}\right)$, where $r \neq r_{1}, r_{2}, \ldots, r_{i}, r=$ $2,3, \ldots, n-1$. By Lemma 13 and Theorem 15, the result follows.

Example 23. The number of spanning trees of $Q_{3}$ and $Q_{4}$ is as shown in Figure 1, where $t\left(Q_{3}\right)=(1 / 3 !) 2^{(2-1)} 3^{(3-1)}$ $(2+3)^{(2-1)(3-1)}=75$ and $t\left(Q_{4}\right)=(1 / 4 !) 2^{(2-1)} 3^{(3-1)} 4^{(4-1)}(2+$ $3)^{(2-1)(3-1)}(2+4)^{(2-1)(4-1)}(3+4)^{(3-1)(4-1)}(2+3+$ $4)^{(2-1)(3-1)(4-1)}=1620609272381440$.

\section{The Number of Spanning Trees of the Generalized Boolean $n$-Cube Network}

Definition 24. The generalized Boolean $n$-cube network $B R(r, n)$ can be defined by

$$
B R(r, n)= \begin{cases}C_{r} & \text { for } n=1 \\ B R(r, n-1) \times K_{2} & \text { for } n \geq 2,\end{cases}
$$

where $C_{r}$ is a cycle with $r$ points. One denotes $B R(r, n)=C_{r} \times$ $K_{2} \times \cdots \times K_{2}$.

Setting $E_{n}$ is the $n \times n$ matrix by

$$
\begin{gathered}
E_{n}=\left[\begin{array}{cccccc}
0 & 1 & 0 & 0 & \cdots & 0 \\
0 & 0 & 1 & 0 & \cdots & 0 \\
\vdots & \vdots & \vdots & \vdots & \cdots & \vdots \\
0 & 0 & 0 & 0 & \cdots & 1 \\
1 & 0 & 0 & 0 & \cdots & 0
\end{array}\right], \\
E_{n}^{i}=\left[\begin{array}{ccccccccc}
1 & & & & \\
0 & \cdots & 0 & 0 & 1 & 0 & 0 & \cdots & 0 \\
0 & \cdots & 0 & 0 & 0 & 1 & 0 & \cdots & 0 \\
\vdots & \vdots & \vdots & \vdots & \vdots & \vdots & \vdots & \cdots & \vdots \\
0 & \cdots & 1 & 0 & 0 & 0 & 0 & \cdots & 0 \\
0 & \cdots & 0 & 1 & 0 & 0 & 0 & \cdots & 0
\end{array}\right] .
\end{gathered}
$$

Lemma 25. The eigenvalues of the adjacent matrix $A\left(K_{r}\right)$ are -1 with multiplicity $r-1$ and $r-1$ with multiplicity 1.

Lemma 26 (see [33]). If $B$ is a sequence matrix, $\lambda$ is an eigenvalue of $B$, and $f$ is a polynomial then $f(\lambda)$ is the eigenvalue of $f(B)$.

Lemma 27. The eigenvalues of the adjacent matrix $A\left(C_{n}\right)$ are $2 \cos (2 \pi k / n)$ for $k=0,1,2, \ldots, n-1$. 

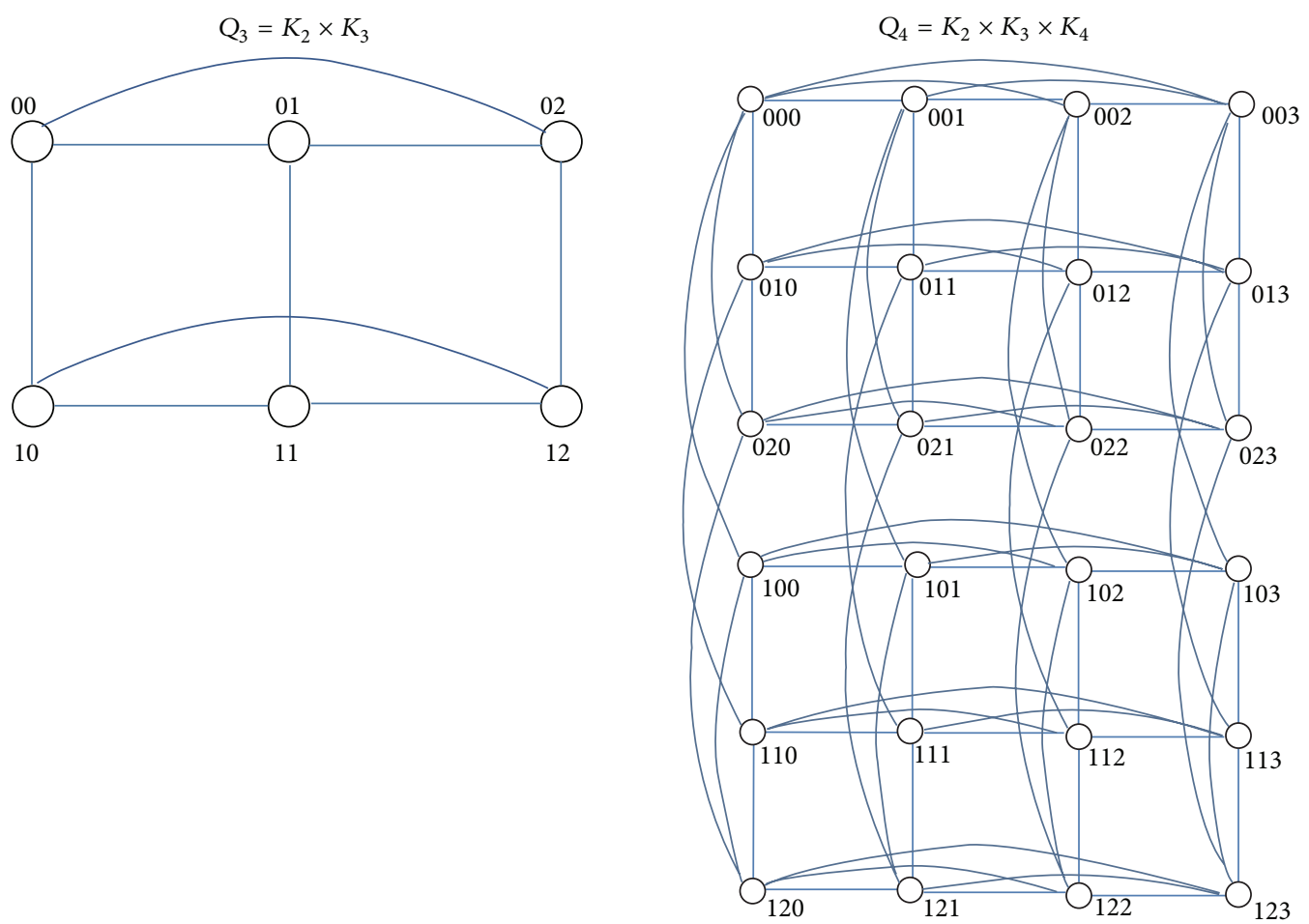

Figure 1

Proof. Since

$$
\begin{aligned}
\operatorname{det}\left(\lambda I_{n}-E_{n}\right) & =\left|\begin{array}{ccccc}
\lambda & -1 & 0 & \cdots & 0 \\
0 & \lambda & -1 & 0 & 0 \\
\vdots & 0 & \ddots & \ddots & 0 \\
0 & \cdots & 0 & \lambda & -1 \\
-1 & 0 & \cdots & 0 & \lambda
\end{array}\right| \\
& =\lambda \cdot \lambda^{n-1}+(-1)(-1)^{n+1}(-1)^{n-1} \\
& =\lambda^{n}-1,
\end{aligned}
$$

the eigenvalues of $E_{n}$ are $e^{(2 \pi k / n) i}$ for $k=0,1,2, \ldots, n-$ 1. It follows that $A\left(C_{n}\right)=E_{n}+E_{n}^{n-1}$. By Lemma 26, the eigenvalues of $A\left(C_{n}\right)$ are $e^{(2 \pi k / n) i}+\left(e^{(2 \pi k / n) i}\right)^{n-1}=e^{(2 \pi k / n) i}+$ $\left(e^{2 \pi k i-(2 \pi k / n) i}\right)=e^{(2 \pi k / n) i}+e^{-(2 \pi k / n) i}=2 \cos (2 \pi k / n)$ for $k=$ $0,1,2, \ldots, n-1$.

The main theorem in [13] can be obtained much more simply as follows.

Theorem 28 (see [13]). The number of spanning trees of $B R(r, n)$ is

$$
\begin{aligned}
t(B R(r, n))= & r 2^{r\left(2^{n-1}-1\right)-n+1} \\
& \times \prod_{i=1}^{n-1}\left(i \prod_{k=1}^{r-1}\left(i+1-\cos \frac{2 \pi k}{r}\right)\right)^{C(n-1, i)} .
\end{aligned}
$$

Proof. It follows that the points of $B R(r, n)$ are $r \cdot 2^{n-1}$ and the degree of any edge of $B R(r, n)$ is $n+1$. By Lemma 25, the eigenvalues of the adjacent matrix $A\left(K_{2}\right)$ are -1 and 1 . By Lemmas 13 and 27, the distinct eigenvalues of the adjacent matrix $A(B R(r, n))$ are

$$
(n-2 i-1)+2 \cos \frac{2 \pi k}{r} \quad k=0,1, \ldots, r-1
$$

with multiplicity $C(n-1, i)$,

where $i=0,1, \ldots, n-1$.

When $k=0$ and $i=0$, the eigenvalue is $n+1$. When $k=0$ and $i=0,1, \ldots, n-1$, the eigenvalues are $(n-2 i-1)+2$. By Theorem 14,

$$
\begin{aligned}
t(B R(r, n)) & \frac{1}{r \cdot 2^{n-1}} \prod_{i=1}^{n-1}((n+1)-((n-2 i-1)+2))^{C(n-1, i)} \\
& \cdot \prod_{k=1}^{r-1} \prod_{i=0}^{n-1}\left((n+1)-\left((n-2 i-1)+2 \cos \frac{2 \pi k}{r}\right)\right)^{C(n-1, i)} \\
= & \frac{1}{r \cdot 2^{n-1}} \prod_{i=1}^{n-1}(2 i)^{C(n-1, i)} \\
& \cdot \prod_{k=1}^{r-1} \prod_{i=0}^{n-1}\left(2\left(i+1-\cos \frac{2 \pi k}{r}\right)\right)^{C(n-1, i)} \cdot
\end{aligned}
$$


Since $\prod_{i=0}^{n-1} 2^{C(n-1, i)}=2^{\sum_{i=0}^{n-1} C(n-1, i)}=2^{2^{n-1}}$ and $\prod_{i=1}^{n-1} 2^{C(n-1, i)}=$ $2^{2^{n-1}-1}$, hence

$$
\begin{aligned}
t(B R(r, n))= & \frac{1}{r \cdot 2^{n-1}} 2^{2^{n-1}-1} 2^{(r-1) 2^{n-1}} \prod_{i=1}^{n-1} i^{C(n-1, i)} \\
& \cdot \prod_{k=1}^{r-1} \prod_{i=0}^{n-1}\left(i+1-\cos \frac{2 \pi k}{r}\right)^{C(n-1, i)} .
\end{aligned}
$$

Since $\prod_{k=1}^{r-1} \sin ^{2}(\pi k / r)=r^{2}$ and $\prod_{k=1}^{r-1}(1-\cos (2 \pi k / r))=$ $\prod_{k=1}^{r-1} 2 \sin ^{2}(\pi k / r)=r^{2} / 2^{r-1}$ as $i=1$,

$$
\begin{aligned}
t(B R(r, n))= & r 2^{r\left(2^{n-1}-1\right)-n+1} \prod_{i=1}^{n-1}{ }_{i} C(n-1, i) \\
& \cdot \prod_{k=1}^{r-1} \prod_{i=1}^{n-1}\left(i+1-\cos \frac{2 \pi k}{r}\right)^{C(n-1, i)} \\
= & r 2^{r\left(2^{n-1}-1\right)-n+1} \\
& \times \prod_{i=1}^{n-1}\left(i \prod_{k=1}^{r-1}\left(i+1-\cos \frac{2 \pi k}{r}\right)\right)^{C(n-1, i)} .
\end{aligned}
$$

\section{The Number of Spanning Trees of the Hypercube Network}

Definition 29. The hypercube network $H(r, m)$ can be defined by

$$
H(r, m)= \begin{cases}C_{r} & \text { for } m=1 \\ H(r, m-1) \times C_{r} & \text { for } m \geq 2,\end{cases}
$$

where $C_{r}$ is a cycle with $r$ points.

Theorem 30. The number of spanning trees of $H(r, m)$ is

$$
t(H(r, m))=\frac{2^{r^{m}-1}}{r^{m}} \prod_{l_{1}, l_{2}, \ldots, l_{m}}\left(m-\sum_{i=1}^{m} \cos \frac{2 \pi l_{i}}{r}\right),
$$

where $l_{1}, l_{2}, \ldots, l_{m}$ satisfy $\left(l_{1}, l_{2}, \ldots, l_{m}\right) \in\{0,1, \ldots, r-1\} \times$ $\{0,1, \ldots, r-1\} \times \cdots \times\{0,1, \ldots, r-1\}-\{(0,0, \ldots, 0)\}$.

Proof. It follows that the points of $H(r, m)$ are $r^{m}$ and the degree of any edge of $H(r, m)$ is $2 m$. By Lemma 26 and Theorem 14,

$$
\begin{aligned}
t(H(r, m)) & =\frac{1}{r^{m}} \prod_{l_{1}, l_{2}, \ldots, l_{m}}\left(2 m-2 \sum_{i=1}^{m} \cos \frac{2 \pi l_{i}}{r}\right) \\
& =\frac{2^{r^{m}-1}}{r^{m}} \prod_{l_{1}, l_{2}, \ldots, l_{m}}\left(m-\sum_{i=1}^{m} \cos \frac{2 \pi l_{i}}{r}\right),
\end{aligned}
$$

where $l_{1}, l_{2}, \ldots, l_{m}$ satisfy $\left(l_{1}, l_{2}, \ldots, l_{m}\right) \in\{0,1, \ldots, r-1\} \times$ $\{0,1, \ldots, r-1\} \times \cdots \times\{0,1, \ldots, r-1\}-\{(0,0, \ldots, 0)\}$.

\section{Conclusion}

Due to the high dependence of the network design and reliability problem, electrical circuits designing issue are on the graph theory. For example, the larger degree of points a network has, the more I/O ports and edges are needed and the more cost is required. The evaluation of this number not only is interesting from a mathematical (computational) perspective but also is an important issue on practical applications. However, the study for spanning trees of the Cartesian product of regular graphs remains an open and important invariant. In this paper, the eigenvalues of the Kirchhoff matrix of Cartesian product of two regular graphs, $G_{1}$ and $G_{2}$, are given by $\lambda+\gamma$ as $\lambda$ and $\gamma$ vary through the eigenvalues of the Kirchhoff matrices $H\left(G_{1}\right)$ and $H\left(G_{2}\right)$, respectively. By this result, the formula for the number of spanning trees of the four regular networks can be simply obtained. Using this formula, the main results in $[13,17,18]$ can be obtained much more simply and will be extended.

\section{Conflict of Interests}

The authors declare that there is no conflict of interests regarding the publication of this paper.

\section{Acknowledgment}

The authors would like to thank the anonymous reviewers for their valuable suggestions.

\section{References}

[1] W.-S. Chiue and B.-S. Shieh, "On connectivity of the Cartesian product of two graphs," Applied Mathematics and Computation, vol. 102, no. 2-3, pp. 129-137, 1999.

[2] J. Jerebic and S. Klavžar, "The distinguishing chromatic number of Cartesian products of two complete graphs," Discrete Mathematics, vol. 310, no. 12, pp. 1715-1720, 2010.

[3] W.-H. Lin and G. J. Chang, "Equitable colorings of Cartesian products of graphs," Discrete Applied Mathematics, vol. 160, no. 3, pp. 239-247, 2012.

[4] B.-S. Shieh, "Super edge- and point-connectivities of the Cartesian product of regular graphs," Networks, vol. 40, no. 2, pp. 9196, 2002.

[5] J.-M. Xu and C. Yang, "Connectivity of Cartesian product graphs," Discrete Mathematics, vol. 306, no. 1, pp. 159-165, 2006.

[6] S. N. Daoud, "Complexity of products of some complete and complete bipartite graphs," Journal of Applied Mathematics, vol. 2013, Article ID 673270, 25 pages, 2013.

[7] J. C. Bermond, F. Comellas, and D. F. Hsu, "Distributed loop computer networks: a survey," Journal of Parallel and Distributed Computing, vol. 24, no. 1, pp. 2-10, 1995.

[8] D. M. Cvetković, M. Doob, and H. Sachs, Spectra of Graphs: Theory and Applications, Johann Ambrosius Barth, Heidelberg, Germany, 3rd edition, 1995.

[9] F. Harary, Graph Theory, Addison-Wesley Publishing, Reading, Mass, USA, 1969.

[10] A. K. Kelmans, "Connectivity of probabilistic networks," Automation and Remote Control, vol. 1967, no. 3, pp. 444-460, 1967. 
[11] F. T. Boesch and H. Prodinger, "Spanning tree formulas and Chebyshev polynomials," Graphs and Combinatorics, vol. 2, no. 3, pp. 191-200, 1986.

[12] A. García, M. Noy, and J. Tejel, "The asymptotic number of spanning trees in $d$-dimensional square lattices," Journal of Combinatorial Mathematics and Combinatorial Computing, vol. 44, pp. 109-113, 2003.

[13] T. C. Huang, J.-F. Wang, C. S. Yang, and J. Y. Lee, "The number of spanning trees of the generalized Boolean $n$-cube network," Computers \& Mathematics with Applications, vol. 16, no. 9, pp. 715-725, 1988.

[14] Z. Lonc, K. Parol, and J. M. Wojciechowski, "On the number of spanning trees in directed circulant graphs," Networks, vol. 37, no. 3, pp. 129-133, 2001.

[15] S. D. Nikolopoulos and P. Rondogiannis, "On the number of spanning trees of multi-star related graphs," Information Processing Letters, vol. 65, no. 4, pp. 183-188, 1998.

[16] W.-M. Yan, W. Myrvold, and K.-L. Chung, "A formula for the number of spanning trees of a multi-star related graph," Information Processing Letters, vol. 68, no. 6, pp. 295-298, 1998.

[17] C. Y. Yang, J. F. Wang, and J. Y. Lee, “The number of spanning trees of the regular networks," International Journal of Computer Mathematics, vol. 23, pp. 185-200, 1988.

[18] C. S. Yang, J. F. Wang, J. Y. Lee, and F. T. Boesch, "Graph theoretic reliability analysis for the Boolean $n$ cube networks," IEEE Transactions on Circuits and Systems, vol. 35, no. 9, pp. 1175-1179, 1988.

[19] X.-R. Yong and T. Acenjian, "The numbers of spanning trees of the cubic cycle $C_{N}^{3}$ and the quadruple cycle $C_{N}^{4}$," Discrete Mathematics, vol. 169, no. 1-3, pp. 293-298, 1997.

[20] Y. Zhang, X. Yong, and M. J. Golin, "The number of spanning trees in circulant graphs," Discrete Mathematics, vol. 223, no. 13, pp. 337-350, 2000.

[21] T. Atajan, N. Otsuka, and X. Yong, "Counting the number of spanning trees in a class of double fixed-step loop networks," Applied Mathematics Letters, vol. 23, no. 3, pp. 291-298, 2010.

[22] T. Atajan, X. Yong, and H. Inaba, "Further analysis of the number of spanning trees in circulant graphs," Discrete Mathematics, vol. 306, no. 22, pp. 2817-2827, 2006.

[23] X. Chen, "The number of spanning trees in directed circulant graphs with non-fixed jumps," Discrete Mathematics, vol. 307, no. 15, pp. 1873-1880, 2007.

[24] X. Chen, Q. Lin, and F. Zhang, "The number of spanning trees in odd valent circulant graphs," Discrete Mathematics, vol. 282, no. 1-3, pp. 69-79, 2004.

[25] Y. Zhang, X. Yong, and M. J. Golin, "Chebyshev polynomials and spanning tree formulas for circulant and related graphs," Discrete Mathematics, vol. 298, no. 1-3, pp. 334-364, 2005.

[26] S. D. Nikolopoulos, L. Palios, and C. Papadopoulos, "Maximizing the number of spanning trees in $K_{n}$-complements of asteroidal graphs," Discrete Mathematics, vol. 309, no. 10, pp. 3049-3060, 2009.

[27] S. D. Nikolopoulos and C. Papadopoulos, "The number of spanning trees in $K_{n}$-complements of quasi-threshold graphs," Graphs and Combinatorics, vol. 20, no. 3, pp. 383-397, 2004.

[28] X. Yong, Y. Zhang, and M. J. Golin, "The number of spanning trees in a class of double fixed-step loop networks," Networks, vol. 52, no. 2, pp. 69-77, 2008.

[29] Y. Zhang, X. Yong, and M. J. Golin, "Chebyshev polynomials and spanning tree formulas for circulant and related graphs," Discrete Mathematics, vol. 298, no. 1-3, pp. 334-364, 2005.
[30] G. Kirchhoff, "Uber die auflosung der gleichurgen, auf welcheman bei der untersn chung der lineren verteilung galvanischer strome gefuhrt wird," Annual Review of Physical Chemistry, vol. 72, pp. 497-508, 1947.

[31] A. K. Kelmans and V. M. Chelnokov, "A certain polynomial of a graph and graphs with an extremal number of trees," Journal of Combinatorial Theory B, vol. 16, pp. 197-214, 1974.

[32] H. Eves, Elementary Matrix Theory, Dover Publications, New York, NY, USA, 1980.

[33] G. E. Shilov, Linear Algebra, Prentice-Hall, Reading, Mass, USA, 1971. 


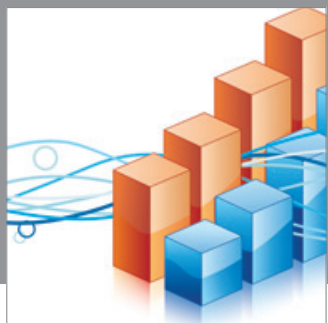

Advances in

Operations Research

mansans

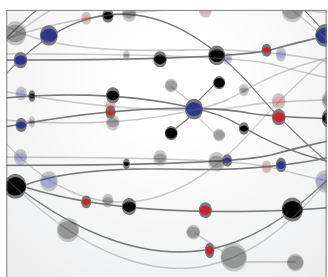

The Scientific World Journal
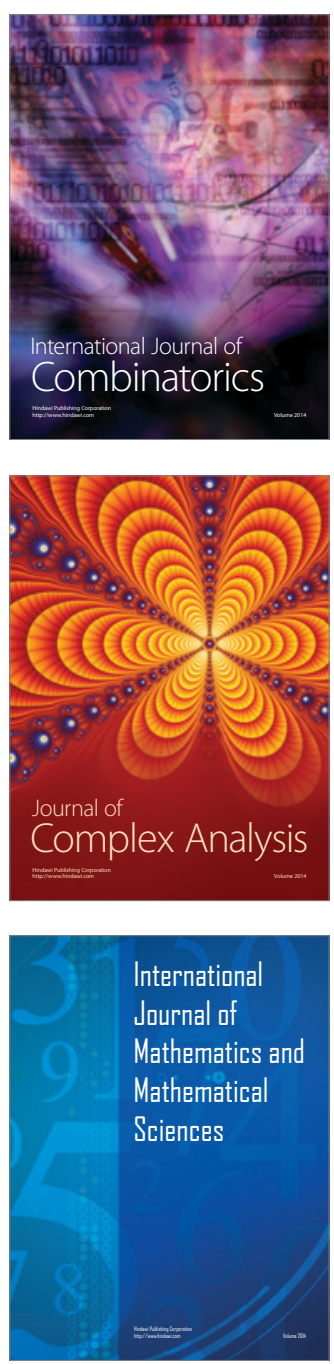
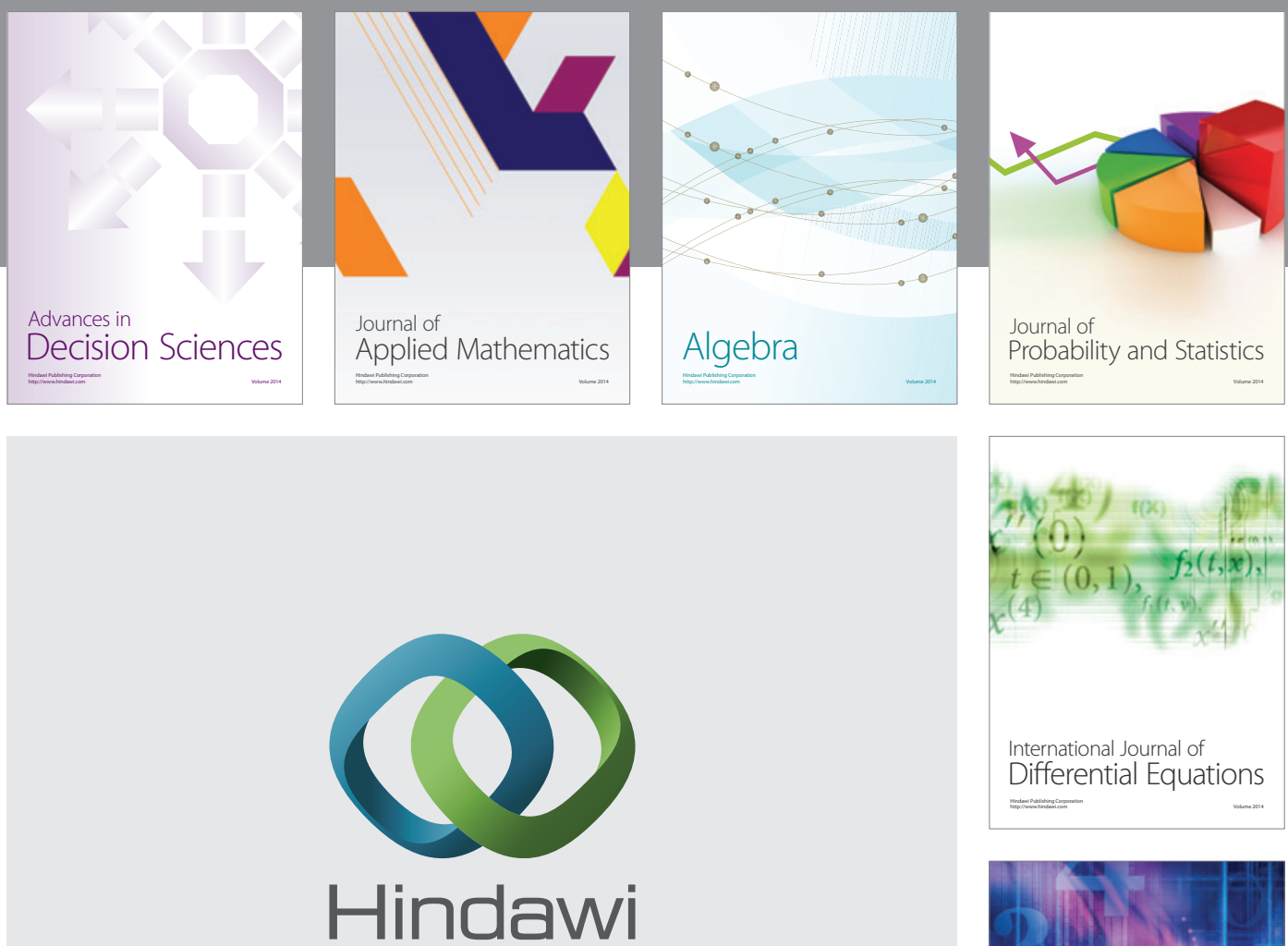

Submit your manuscripts at http://www.hindawi.com
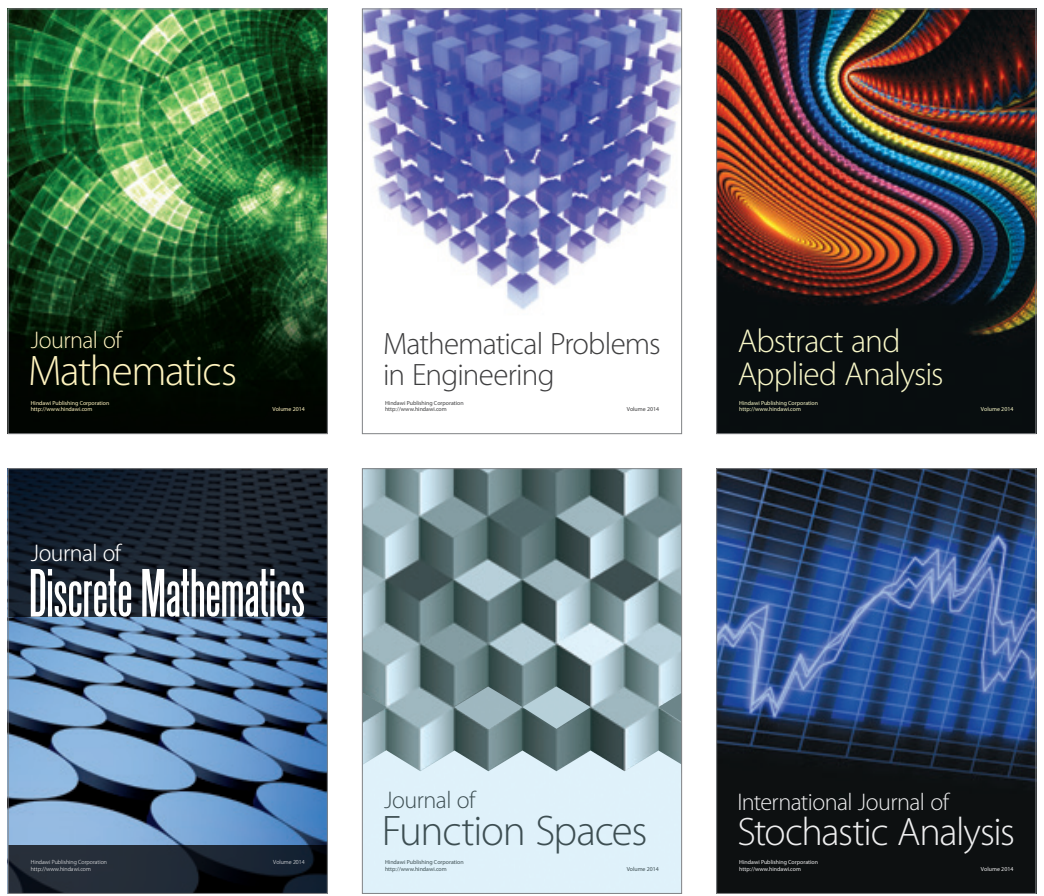

Journal of

Function Spaces

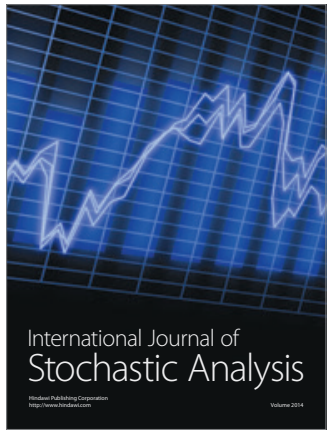

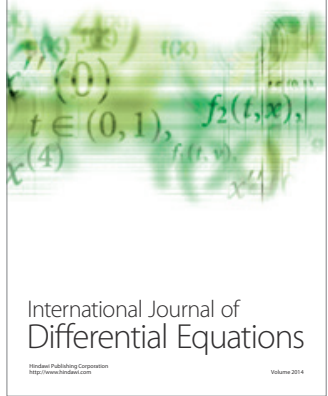
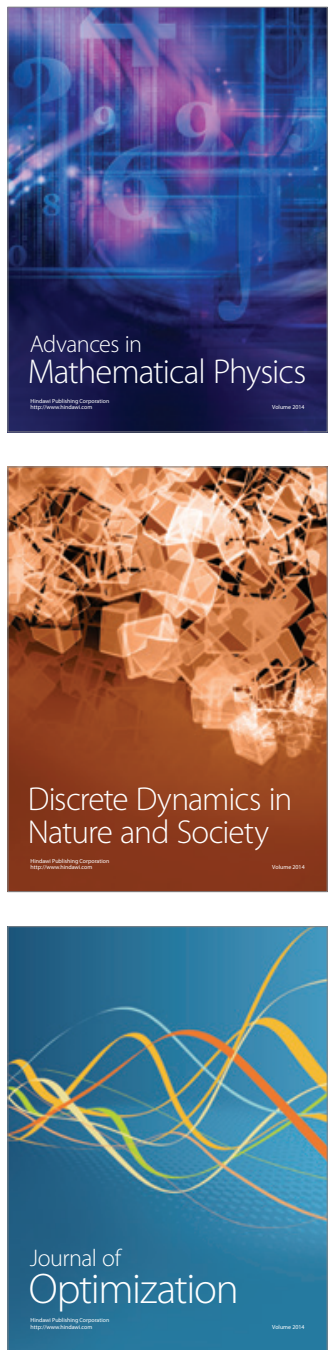